\title{
Efectos de un programa de educación para la convivencia en factores del desarrollo socio-emocional y creativo en niños y niñas de 7 a 11 años
}

\section{Effects of an education program for coexistence on creative and socio-emotional development factors for children aged 7-11 years}

\author{
José Ignacio Pérez ${ }^{1} \quad$ Maite Garaigordobil \\ Zuriñe Adrada Leire de Miguel \\ Universidad del País Vasco, España
}

(Rec: Marzo 2011 - Acep: Octubre 2011)

\begin{abstract}
Resumen
El estudio se plantea tres objetivos: (1) analizar los efectos de un programa de educación para la convivencia implementado con niños y niñas de 7 a 9 años, durante 3 años, en factores del desarrollo socio-emocional y creativo; (2) explorar diferencias de género en dichos factores, y (3) determinar si el género afecta a los efectos de la intervención. Se utilizó un diseño experimental pretest-intervención-postest con grupo de control. La muestra consistió en 80 participantes de 7 a 9 años (53 experimentales, 27 control). En la fase pretest y postest se administraron 5 instrumentos de evaluación. Los resultados de los análisis de varianza sugieren que el programa incrementó significativamente diversas conductas facilitadoras de la socialización, la elección de compañeros de juego, el número de compañeros percibidos como prosociales y las conductas-rasgos de personalidad creadora. Los resultados confirman que la intervención afectó de forma similar a ambos sexos.
\end{abstract}

Palabras clave: evaluación de programas, desarrollo infantil, diferencias de género.

\begin{abstract}
The purpose of this study is three-fold: (1) to assess the effects of a three-year educational programme for coexistence with children aged 7 to 9 on socio-emotional and creative factors, (2) to identify gender differences in these factors, and (3) to analyze whether gender has an influence on the effects of the programme. The experimental design was pretest-intervention-posttest with control group, with a sample made up of 80 participants aged 7 to 9 years ( 53 experimental and 27 control). Five assessment instruments were applied before and after the program. The results of the analyses of variance suggest that de program significantly increased different behaviours which facilitate socialization, play-mate election, number of classmates considered prosocial, creative behaviour and creative personality traits. Gender did not influence the effects of the intervention.
\end{abstract}

Key words: program evaluation, children development, gender differences.

\footnotetext{
Correspondencia a: José Ignacio Pérez. Dirección: Facultad de Psicología, Universidad del País Vasco, Avenida de Tolosa 70, 20018 Donostia-San Sebastián. Tel: 34- 943015641 / Fax: 34-943 015670. E-mail: joseignacio.perez@ehu.es.
} 


\section{Introducción}

En los últimos años se está produciendo un cambio significativo en la concepción de la educación. Durante mucho tiempo la escuela se ha considerado una institución fuertemente vinculada al éxito académico, sin embargo, hoy en día se está haciendo un esfuerzo por superar esta concepción de la escuela como mero agente transmisor de conocimiento, en favor de una ampliación de los procesos educativos que incluya la educación en los valores y actitudes que necesita una convivencia pacífica; así pues, la escuela está progresivamente abandonando este enfoque puramente individualista e intelectualista, intentando integrar en su actividad cotidiana la formación de personas solidarias, tolerantes, pacíficas...

Progresivamente se está enfatizando la importancia de que la escuela consiga que los alumnos convivan con sus compañeros y con los adultos, de tal modo que les sirva de aprendizaje para la convivencia en sociedad. Así, se subraya la necesidad de realizar un trabajo sistematizado desde los centros educativos para promover una convivencia pacífica. La sociedad emplaza a la escuela a educar para la convivencia y la paz, a fomentar la educación en valores ético-morales, a educar en el respeto a los derechos humanos, la interculturalidad.... Sin embargo, pese al consenso existente a este respecto entre los profesionales de la psicología y la educación, son escasos los centros educativos que llevan a cabo estas experiencias como parte del proyecto educativo del centro, y no abundan los programas de intervención validados con estudios metodológicamente rigurosos que permitan a profesores y psicólogos implementar este tipo de experiencias (Garaigordobil \& Fagoaga, 2006).

Desde finales de la década de los ochenta, un grupo de profesionales de la psicología y la educación han estado trabajando en este ámbito de intervención, diseñando, aplicando y validando experimentalmente programas para grupos de niños y niñas y adolescentes, con la finalidad de estimular el desarrollo socioemocional y creativo, fomentando variables tales como la comunicación, las relaciones de ayuda y confianza, la capacidad de cooperación grupal, la expresión emocional, el respeto por las diferencias, la aceptación del otro... La evaluación experimental de estos programas de intervención psicoeducativa basados en actividades cooperativas y creativas ha evidenciado su efectividad en el desarrollo socio-emocional y creativo infantil. En concreto, en el ámbito de educación primaria se ha observado que el desarrollo de actividades cooperativas y creativas en esta etapa evolutiva favorece: (1) un incremento de conductas sociales positivas (liderazgo, jovialidad, sensibilidad social, respeto-autocontrol), y una disminución de conductas sociales negativas (agresividad, apatía-retraimiento, ansiedad-timidez); (2) una mejora de la imagen de los compañeros del grupo, que son menos rechazados y percibidos como más prosociales y creativos; (3) un mayor nivel de estabilidad emocional; (4) una mejora del autoconcepto; y (5) un incremento de conductas y rasgos de personalidad creadora (Garaigordobil 2003ab, 2004ab, 2005ab; Garaigordobil \& Fagoaga, 2006; Garaigordobil \& Pérez, 2004).

En este contexto cabe resaltar que en los últimos años el problema de la violencia juvenil ha sido objeto de creciente preocupación por parte de los profesionales de la educación y de la salud mental en todo el mundo. En consecuencia, una línea de investigación se ha centrado en el diseño y la evaluación de programas para la prevención y disminución de la violencia. Los resultados de estos estudios han puesto de manifiesto la eficacia de este tipo de intervenciones llevadas a cabo en contextos educativos, clínicos y comunitarios (Chandy, 2007; Dawn \& Shaughnessy, 2005; Dole, 2006; Farrell, Meyer \& White 2001; Freiden, 2006; Roberts, White, \& Yeomans, 2004; Segawa, Ngwe, Li \& Flay, 2005; Sege, Licenziato \& Webb, 2005; Simón et al., 2008; Slone \& Shoshani, 2008). Con la finalidad de potenciar el desarrollo socio-emocional y prevenir la violencia se efectúa esta investigación en la que se implementa y evalúa un programa de educación para fomentar las conductas prosociales y la convivencia.

Tomando como referencia los mencionados estudios, este trabajo se plantea los siguientes objetivos: (1) analizar los efectos de un programa de educación para la convivencia implementado con niños y niñas de 7 a 9 años, a lo largo de 3 cursos escolares consecutivos, en factores del desarrollo socio-emocional y creativo, tales como conductas sociales en el ambiente escolar, inestabilidad emocional, imagen de los compañeros del grupo, autoconcepto, y conductas y rasgos de personalidad creadora; (2) explorar la existencia de diferencias de género en niños y niñas de estas edades en dichos factores; y (3) determinar si el género afectará a los efectos del programa de educación para la convivencia.

A nivel global, el estudio postula que el programa de educación para la convivencia estimulará el desarrollo socio-emocional y creativo en los niños y niñas de estas edades, proponiendo 7 hipótesis; en concreto, se plantea que el programa: (1) aumentará significativamente las conductas facilitadoras de la socialización (liderazgo, jovialidad, sensibilidad social, respeto-autocontrol) y 
de adaptación social global, elección de compañeros de juego, número de compañeros percibidos como prosociales, autoconcepto, y conductas y rasgos de personalidad creadora; y (2) disminuirá significativamente las conductas perturbadoras de la socialización (agresividad-terquedad, apatía-retraimiento, ansiedadtimidez), inestablidad emocional, rechazo de compañeros de juego y número de compañeros percibidos como no prosociales. Además, se propone que: (3) las niñas obtendrán puntuaciones significativamente superiores en las conductas facilitadoras de la socialización, adaptación social global, elección de compañeros de juego, y número de compañeros percibidos como prosociales; (4) los niños obtendrán puntuaciones significativamente superiores en las conductas perturbadoras de la socialización, inestabilidad emocional, rechazo de compañeros de juego, y número de compañeros percibidos como no prosociales; (5) no se observarán diferencias de género en el autoconcepto; (6) no se evidenciarán diferencias de género en conductas-rasgos de personalidad creadora; y (7) el género no afectará a los efectos del programa de educación para la convivencia.

\section{Método}

\section{Participantes}

La muestra está constituida por 80 participantes de 7 a 9 años de edad al inicio del programa ( 9 a 11 a la finalización del mismo), distribuidos en 6 grupos pertenecientes a 2 centros escolares públicos del País Vasco (España). Del conjunto de la muestra, 53 sujetos fueron asignados aleatoriamente a la condición experimental (4 grupos), mientras que 27 desempeñaron la condición de control (2 grupos). La muestra, inicialmente constituida por 92 participantes (62 experimentales y 30 de control), se redujo a 80 tras los tres años de implementación del programa (repetidores, cambios de centro). Tal y como puede observarse en la Tabla 1, la mitad del total de los participantes se encontraban al inicio del programa en $2^{\circ}$ curso de Educación Primaria, y la otra mitad en $3^{\circ}$, siendo el $47.5 \%$ niños y el $52.5 \%$ niñas. Los sujetos pertenecen a un ámbito económico y cultural medio.

\section{Diseño y procedimiento}

El estudio empleó una metodología experimental, en concreto un diseño de medidas repetidas pretestintervención-postest con grupo de control. En la fase pretest, durante las primeras semanas del primer curso escolar en el que se implementó el programa (septiembre), se administraron 5 instrumentos de evaluación para medir las variables dependientes sobre las que se hipotetizó que el programa iba a tener efectos positivos. Posteriormente, los participantes experimentales realizaron el programa de intervención a lo largo de tres cursos escolares, que consistió en una sesión semanal de tutoría (45 minutos) y otra de plástica (90 minutos) durante los dos primeros cursos, y una sesión conjunta de tutoría y plástica (90 minutos) durante el tercer curso. Las sesiones fueron dirigidas por los profesores tutores de los grupos experimentales, quienes recibieron los materiales, la formación y el apoyo pedagógico continuo de los responsables del programa. A lo largo de estos tres cursos escolares, los participantes de control realizaron las tutorías y las actividades curriculares de plástica del programa del centro escolar, con lo que se evitó el efecto Hawthorne, ya que recibieron otro tipo de instrucción y el mismo nivel de atención. En la fase postest, durante las últimas semanas del tercer y último curso en el que se llevó a cabo la intervención (junio), se administraron los mismos instrumentos de evaluación que en la fase pretest. La administración de dichos instrumentos también fue llevada a cabo por los profesores tutores de los grupos, formados en seminarios específicos con dicha finalidad. No obstante, la corrección de las pruebas fue ciega y ejecutada por profesionales de la psicología ajenos a la implementación del programa.

Tabla 1. Distribución de los participantes experimentales y de control por curso y sexo en la fase pretest.

\begin{tabular}{|c|c|c|c|c|c|c|c|c|c|c|c|c|c|c|}
\hline \multirow[b]{3}{*}{ Curso } & \multicolumn{4}{|c|}{$\begin{array}{l}\text { Experimental } \\
\quad(n=53)\end{array}$} & \multicolumn{4}{|c|}{$\begin{array}{l}\text { Control } \\
(n=27)\end{array}$} & \multicolumn{6}{|c|}{$\begin{array}{c}\text { Total } \\
(n=80)\end{array}$} \\
\hline & \multicolumn{2}{|c|}{ Varón } & \multicolumn{2}{|c|}{ Mujer } & \multicolumn{2}{|c|}{ Varón } & \multicolumn{2}{|c|}{ Mujer } & \multicolumn{2}{|c|}{ Varón } & \multicolumn{2}{|c|}{ Mujer } & \multicolumn{2}{|c|}{ Total } \\
\hline & $n$ & $\%$ & $n$ & $\%$ & $n$ & $\%$ & $n$ & $\%$ & $n$ & $\%$ & $n$ & $\%$ & $n$ & $\%$ \\
\hline $2^{\circ}$ & 10 & $40 \%$ & 15 & $60 \%$ & 9 & $60 \%$ & 6 & $40 \%$ & 19 & $47.5 \%$ & 21 & $52.5 \%$ & 40 & $50 \%$ \\
\hline $3^{\circ}$ & 11 & $39.3 \%$ & 17 & $60.7 \%$ & 8 & $66.7 \%$ & 4 & $33.3 \%$ & 19 & $47.5 \%$ & 21 & $52.5 \%$ & 40 & $50 \%$ \\
\hline Total & 21 & $39.6 \%$ & 32 & $60.3 \%$ & 17 & $63 \%$ & 10 & $37 \%$ & 38 & $47.5 \%$ & 42 & $52.5 \%$ & 80 & $100 \%$ \\
\hline
\end{tabular}


Cuadro 1. Variables dependientes e instrumentos de evaluación pretest-postest.

\begin{tabular}{|c|c|c|c|c|}
\hline Instrumentos & Variables dependientes & Fuente & Aplicación & Tiempo \\
\hline $\begin{array}{l}\text { BAS-1 } \\
\text { Batería de Socialización } \\
\text { (Silva \& Martorell, 1983). }\end{array}$ & $\begin{array}{l}\text { Conductas sociales: } \\
\text { - Liderazgo } \\
\text { - Jovialidad } \\
\text { - Sensibilidad social } \\
\text { - Respeto-autocontrol } \\
\text { - Agresividad-terquedad } \\
\text { - Apatía-retraimiento } \\
\text { - Ansiedad-timidez } \\
\text { - Adaptación social global }\end{array}$ & Profesores/as & Individual & $15^{\prime}$ \\
\hline $\begin{array}{l}\text { DFH } \\
\text { El test del dibujo de la figura humana } \\
\text { (Koppitz, 1976) }\end{array}$ & Inestabilidad emocional. & Niños/as & Colectiva & $20^{\prime}$ \\
\hline $\begin{array}{l}\text { CS } \\
\text { Cuestionario sociométrico: compañero de } \\
\text { juego y prosocial (Moreno, 1934/1972. } \\
\text { Adaptación de Garaigordobil, 2004a) }\end{array}$ & $\begin{array}{l}\text { Imagen de los compañeros: } \\
\text { - Compañeros de juego } \\
\text { - Compañeros prosociales }\end{array}$ & Niños/as & Colectiva & $10^{\prime}$ \\
\hline $\begin{array}{l}\text { LAEA } \\
\text { Listado de adjetivos para la evaluación } \\
\text { del autoconcepto (Garaigordobil, 2003b) }\end{array}$ & Autoconcepto & Niños/as & Colectiva & $10^{\prime}$ \\
\hline $\begin{array}{l}\text { EPC } \\
\text { Escala de personalidad creadora } \\
\text { (Garaigordobil, 2004a) }\end{array}$ & $\begin{array}{l}\text { Conductas y rasgos de perso- } \\
\text { nalidad creadora }\end{array}$ & $\begin{array}{l}\text { Profesores/as } \\
\text { Padres }\end{array}$ & Individual & $10^{\prime}$ \\
\hline
\end{tabular}

\section{Instrumentos de evaluación}

Con la finalidad de medir las variables dependientes se administran 5 instrumentos de evaluación con adecuadas garantías psicométricas (ver Cuadro 1). Además, con el objeto de evaluar la percepción subjetiva del cambio en los sujetos experimentales al finalizar la implementación del programa llevado a cabo durante tres cursos escolares, los 53 niños y niñas implicados en la intervención cumplimentaron un cuestionario de evaluación del programa diseñado "ad hoc" con esa finalidad.

BAS-1. Batería de Socialización (Silva \& Martorell, 1983). Se trata de un cuestionario para evaluar la socialización de niños y adolescentes de 6 a 15 años en ambientes escolares. La tarea consiste en leer las afirmaciones y responder si el contenido del ítem puede ser aplicado al alumno que es evaluado por el profesor. Los 118 ítems de la batería permiten obtener un perfil de socialización con siete escalas, cuatro de ellas relacionadas con conductas sociales positivas (conductas de liderazgo, jovialidad, sensibilidad social y respeto-autocontrol), tres relacionadas con aspectos negativos o perturbadores para la socialización (conductas de agresividad-terquedad, apatía-retraimiento y ansiedad-timidez), y una escala de apreciación global de la socialización.

DFH. El test de dibujo de la figura humana (Koppitz, 1976). Para evaluar la estabilidad emocional se analizan los indicadores emocionales que aparecen en el dibujo de la figura humana. La tarea consiste en realizar el dibujo de una persona. Para la corrección, el análisis se centra en 30 indicadores emocionales, signos objetivos que reflejan actitudes, preocupaciones, relaciones interpersonales no satisfactorias y ansiedades en los niños. Los indicadores emocionales cumplen 3 requisitos: validez clínica, inusuales en los DFH de niños normales, y no relacionados ni con la edad ni con la maduración. En el proceso de corrección, se otorga un punto cuando el indicador aparece en el dibujo. La significación diagnóstica de los indicadores se acrecienta cuando se toma en cuenta su número total. Cuando un dibujo no muestra ningún indicador emocional, parece probable que el niño no tenga problemas emocionales.

CS. Cuestionario sociométrico: compañero de juego y prosocial (Moreno, 1934/1972). En este 
cuestionario cada miembro del grupo indica las personas del grupo -tantas como quiera- a las que elige o rechaza como: 1) compañeros de juego, y 2) compañeros "prosociales" (compañeros que ayudan, comparten, colaboran, consuelan...).

LAEA. Listado de adjetivos para la evaluación del autoconcepto (Garaigordobil, 2003b). Este listado contiene 28 adjetivos de cualidad positiva, incluyendo descripciones de características físicas (3), intelectuales (6), afectivas (11) y de sociabilidad (8), en base a los cuales se evalúa el autoconcepto.

EPC. Escala de personalidad creadora (Garaigordobil, 2004a). Esta prueba contiene 22 afirmaciones o frases con las que se evalúa la creatividad explorando diversas conductas y rasgos características de las personalidades creadoras. Padres y profesores valoran en una escala de estimación de "nada, algo, bastante o mucho" la intensidad con la que se puede aplicar la conducta o el rasgo a los niños y niñas. Algunos ejemplos de ítems de esta escala son: "muestra curiosidad sobre muchas cosas haciendo continuas preguntas sobre variados temas; ofrece soluciones originales a los problemas que observa; siente interés por actividades artísticas como el dibujo, la pintura; le gustan los juegos con las palabras; inventa canciones, poesías, chistes; tiene sentido del humor; es independiente; está abierto a nuevas experiencias...". Para la corrección se puntúa con $0,1,2$ ó 3 puntos respectivamente, en función del grado de intensidad de aplicación del contenido de la frase (nada, algo, bastante, mucho).

\section{Intervención}

El programa de acción tutorial para educación primaria fue diseñado por el equipo pedagógico de Ikertze Proyectos Educativos como un proyecto piloto para la educación en la convivencia, el respeto de los derechos humanos y la cultura de paz. Dicho programa se implementó a lo largo de tres cursos escolares, y consistió en una sesión semanal de tutoría (45 minutos) y otra de plástica (90 minutos) durante los dos primeros cursos, y una sesión conjunta de tutoría y plástica (90 minutos) durante el tercer y último curso. Las sesiones fueron dirigidas por los tutores de los grupos, quienes recibieron los materiales, la formación y el apoyo pedagógico continuo de los responsables del programa.

Dicho programa se estructura en torno a los derechos de los niños y las niñas recogidos en la Convención de los Derechos del Niño (UNICEF 2006/1989), y utiliza diversas actividades cooperativas y creativas que tienen como principales objetivos estimular: (1) la curiosidad, la capacidad de hacer preguntas, la capacidad de escucha y de observación; (2) la expresión de emociones a través de la palabra, el dibujo, la pintura, la dramatización... (3) los procesos de comunicación dentro del grupo (exponer, escuchar, dialogar, negociar, tomar decisiones por consenso...); (4) las interacciones prosociales (ayudar, cooperar, compartir, consolar...); y (5) el empleo de diversas técnicas, materiales y soportes comunicativos para abordar distintos conceptos relacionados con los derechos de los niños y las niñas (derecho a la identidad, al desarrollo, a la vida y a la participación).

El programa se estructura en tres niveles, uno por cada uno de los cursos en los que se implementa. El eje conceptual son los derechos de la infancia (un derecho en cada nivel, y el derecho a la participación como tema transversal): "Encuentro de identidades", "Derecho al desarrollo" y "Derecho a la vida: convivencia y conflicto". Las herramientas principales que se utilizan para el desarrollo de las actividades son el arte, y la reflexión y el diálogo sobre lo realizado.

El objetivo general del primer nivel del programa, "Encuentro de identidades", es el de tomar conciencia de nuestra identidad -individual, común y planetariadesde el respeto a uno mismo y a la condición humana. En concreto, se plantea contribuir a reconocernos como: (1) personas únicas; (2) que pertenecemos a una especie y que con otras compartimos un planeta que nos es imprescindible para vivir; (3) que nacemos en una familia, en un sitio, en una comunidad determinada que nos trasmite su idioma, sus costumbres, sus creencias, su cultura... y que compartimos el planeta con otras personas, otros pueblos con los que nos podemos sentir iguales y diferentes; (4) que estamos vivos y por tanto con capacidad de aprender y de enseñar, de dar y recibir; (5) con pensamientos, emociones y energía para vivir; (6) con un cuerpo que nos acompaña, nos cuida y podemos cuidar; y (7) con sueños, deseos, derechos y deberes.

El segundo nivel del programa, "Derecho al desarrollo", se plantea como objetivo general acercar a los niños y niñas el concepto del desarrollo como un derecho que implica a su vez responsabilidades. Las actividades de este nivel del programa tienen como principales objetivos: (1) tomar conciencia del desarrollo personal como el viaje de nuestra vida en sus diferentes etapas (el nacimiento, la niñez, la juventud, la edad adulta y la vejez), con momentos difíciles y momentos tranquilos, con imprevistos...; (2) entender que las personas nos vamos desarrollando a medida que pasa el tiempo y observar que en este crecimiento se desarrolla nuestro cuerpo, nuestras emociones y nuestro intelecto; (3) tomar 
conciencia de que para que nuestro cuerpo crezca y se desarrolle de forma saludable necesita nuestro cuidado (alimentación, hábitos saludables...); (4) ser capaces de reconocer distintas emociones, expresarlas, dialogar sobre aquello que las provoca y sus posibles consecuencias (alegría, miedo, rabia, confianza, envidia...); y (5) reflexionar sobre nuestras habilidades de pensamiento y sobre lo que los maestros y las maestras aportan y ayudan a su desarrollo.

Por último, en el tercer nivel del programa, "Derecho a la vida: convivencia y conflicto", se aborda el concepto de vida y se subrayan las diferencias entre la vida de los seres humanos y la de los animales y las plantas. En concreto, se pretende: (1) introducir el concepto de convivencia, profundizando en las actitudes y sucesos que favorecen y dificultan la convivencia entre las personas; (2) reflexionar sobre los prejuicios y su influencia en nuestra vida; (3) diferenciar catástrofe de conflicto, para abordar este último desde una perspectiva positiva, como algo natural y que tiene solución; y (4) favorecer la capacidad de analizar los conflictos y las distintas actitudes que pueden surgir frente a ellos para comprenderlos mejor y poder resolverlos de manera más adecuada.

Con la finalidad de clarificar las características del programa, se presenta a modo de ejemplo la ficha técnica de una de las actividades desarrolladas en el programa (ver Cuadro 2).

\section{Resultados}

\section{Efectos del programa en factores del desarrollo socio-emocional y creativo}

Tras verificar los supuestos básicos (normalidad, homogeneidad de varianza e independencia), con los datos obtenidos en las pruebas pretest-postest se

Cuadro 2. Ficha técnica de la actividad "Un árbol de posibilidades" (Nivel I: Encuentro de identidades).

\section{UN ÁRBOL DE POSIBILIDADES}

Temporalización: Dos sesiones de tutoría y dos de plástica.

Objetivos: Que los niños y niñas desarrollen una imagen positiva de ellos mismos y de sus compañeros, tomando conciencia de: (a) lo que podemos hacer, y (b) lo que podemos llegar a hacer.

\section{Descripción de la actividad:}

Plástica (45 minutos)

Pedimos a los niños que dibujen un árbol y observamos juntos los resultados de todos (si se parecen o no, qué tienen en común; introducimos el concepto de forma y estereotipo). A continuación les mostramos fotografías de árboles distintos, diferenciando claramente el tronco de las ramas. Introducimos la regla del juego que nos servirá para que cada uno construya un nuevo árbol: "de cada rama salen las ramas que quieras, pero cada una es más fina que la anterior".

- Trabajo individual: Disponemos de folios y rotuladores de diferentes grosores o de tiras de cartulinas de anchuras diferentes y construimos nuestros árboles. Podemos cambiar el soporte, modificarlo, elaborarlo con dos cartulinas... variar los recursos para favorecer la creatividad.

- Trabajo colectivo: Disponemos de papel de embalar y cartulinas grandes para construir, entre todos, nuestro gran árbol de las posibilidades. Una vez hecho el árbol, cada niño colocará su nombre y fotografía en una rama.

Tutoría (45 minutos)

Hablamos sobre nuestras capacidades; pedimos a los niños y niñas que piensen en algo que no son capaces de hacer. Podemos reflexionar sobre si las respuestas que han dado son cosas imposibles hacer, o simplemente no tienen la capacidad de hacerlas ahora. Pensamos también en nuestras capacidades y hacemos una lista o numeramos las cosas que podemos hacer.

Plástica (45 minutos)

Hacemos en papeles diferentes varias hojas para nuestro árbol. Tienen que ser grandes porque luego escribiremos en ellas nuestras capacidades. Pasaremos a ordenador las cosas que podemos hacer (sobre las que hablamos en tutoría), y cada niño coloca el texto sobre las hojas que hemos hecho para nuestras ramas (por ejemplo, yo soy capaz de nadar, de patinar, de saltar la cuerda...). Las cosas que todavía no podemos hacer y que nos gustaría conseguir, las podemos escribir en Post-it que pegaremos en el tronco del árbol y que cuando las vayamos consiguiendo se convertirán en hojas. Los niños y niñas pueden también dibujar hojas en las ramas de sus compañeros y escribir capacidades de ellos que aprecien.

Tutoría (45 minutos)

Debatimos entre todos qué cosas nos pueden ayudar a realizar lo que queremos conseguir, y cómo podemos ayudar a nuestros compañeros para que consigan lo que se proponen. Dejaremos el árbol en clase, y de vez en cuando le daremos un repaso para ver cómo van esas cosas que queremos conseguir (apuntadas en Post-it).

Material necesario: Papel de embalar, rotuladores de diferentes colores y grosores, cartulinas base, tiras de cartulinas de colores, fotografías de árboles diferentes, pegamento, tijeras, fotografías de los propios niños y niñas, Post-it. 
calcularon las medias y las desviaciones típicas en relación a cada variable objeto de estudio, para los participantes experimentales y control, llevándose a cabo análisis de varianza multivariados y univariados (MANOVAs, ANOVAs) con las puntuaciones pretest, postest, así como con las diferencias pretest-postest, y obteniendo las covarianzas (MANCOVAs, ANCOVAs) en aquellas variables en las que se encontraron diferencias significativas entre ambas condiciones en la fase pretest. Los resultados se presentan en la Tabla 2.

Tabla 2. Medias, desviaciones típicas y resultados del análisis de varianza de experimentales y control en variables socioemocionales y de creatividad en la fase pretest, postest, y en la diferencia pretest-postest.

\begin{tabular}{|c|c|c|c|c|c|c|c|c|c|c|c|c|c|c|c|}
\hline \multirow[b]{3}{*}{ Variables } & \multicolumn{6}{|c|}{$\begin{array}{l}\text { Grupo Experimental } \\
\qquad(n=53)\end{array}$} & \multicolumn{6}{|c|}{$\begin{array}{l}\text { Grupo Control } \\
\quad(n=27)\end{array}$} & \multicolumn{3}{|c|}{$\begin{array}{l}\text { Experimental-Control } \\
\qquad(n=80)\end{array}$} \\
\hline & \multicolumn{2}{|c|}{$\begin{array}{l}\text { Evaluación } \\
\text { Pre }\end{array}$} & \multicolumn{2}{|c|}{$\begin{array}{l}\text { Evaluación } \\
\text { Post }\end{array}$} & \multicolumn{2}{|c|}{$\begin{array}{l}\text { Diferencia } \\
\text { Pre-Post }\end{array}$} & \multicolumn{2}{|c|}{$\begin{array}{l}\text { Evaluación } \\
\text { Pre }\end{array}$} & \multicolumn{2}{|c|}{$\begin{array}{l}\text { Evaluación } \\
\text { Post }\end{array}$} & \multicolumn{2}{|c|}{$\begin{array}{l}\text { Diferencia } \\
\text { Pre-Post }\end{array}$} & \multirow{2}{*}{$\begin{array}{c}\begin{array}{c}\text { Anova } \\
\text { Pre }\end{array} \\
F(1,78)\end{array}$} & \multirow{2}{*}{$\begin{array}{l}\text { Anova } \\
\text { Pre-Post } \\
F(1,78)\end{array}$} & \multirow{2}{*}{$\begin{array}{l}\text { Ancova } \\
\text { Pre-Post } \\
F(1,78)\end{array}$} \\
\hline & M & DT & M & DT & M & $D T$ & $M$ & $D T$ & $M$ & $D T$ & $M$ & $D T$ & & & \\
\hline Liderazgo & 23.06 & 10.12 & 29.49 & 12.53 & 6.43 & 10.30 & 26.41 & 14.34 & 28.04 & 10.12 & 1.63 & 13.17 & 1.46 & 3.20 & 1.84 \\
\hline Jovialidad & 23.72 & 6.82 & 26.32 & 7.31 & 2.60 & 7.58 & 26.63 & 8.22 & 24.74 & 7.18 & -1.89 & 6.77 & 2.83 & $6.73^{*}$ & $3.88+$ \\
\hline $\begin{array}{l}\text { Sensibilidad } \\
\text { social }\end{array}$ & 21.21 & 7.18 & 25.51 & 7.79 & 4.30 & 8.87 & 25.48 & 12.43 & 23.07 & 6.58 & -2.41 & 11.97 & 3.79 & $8.03^{* *}$ & $3.99^{*}$ \\
\hline $\begin{array}{l}\text { Respeto- } \\
\text { autocontrol }\end{array}$ & 34.11 & 13.34 & 39.32 & 10.45 & 5.21 & 11.51 & 39.89 & 12.94 & 38.04 & 10.87 & -1.85 & 13.03 & 3.41 & $6.14^{*}$ & 2.67 \\
\hline $\begin{array}{l}\text { Agresividad- } \\
\text { terquedad }\end{array}$ & 9.02 & 9.28 & 6.02 & 6.44 & -3.00 & 6.67 & 2.81 & 3.61 & 4.15 & 6.13 & 1.33 & 5.07 & $11.12^{* * * *}$ & $8.78^{* * *}$ & 1.15 \\
\hline $\begin{array}{l}\text { Apatía- } \\
\text { retraimiento }\end{array}$ & 6.26 & 7.94 & 5.45 & 6.50 & -0.81 & 6.95 & 5.93 & 9.29 & 6.85 & 8.88 & 0.93 & 6.86 & 0.29 & 1.12 & 1.32 \\
\hline $\begin{array}{l}\text { Ansiedad- } \\
\text { timidez }\end{array}$ & 9.98 & 5.05 & 7.40 & 5.49 & -2.58 & 5.90 & 5.93 & 4.44 & 9.56 & 4.57 & 3.63 & 6.91 & $14.45^{* * *}$ & $17.62^{* * * *}$ & $5.88 *$ \\
\hline $\begin{array}{l}\text { Adaptación } \\
\text { social global }\end{array}$ & 32.89 & 7.05 & 35.66 & 6.81 & 2.77 & 6.23 & 35.07 & 8.26 & 35.22 & 7.51 & 0.15 & 7.75 & 1.53 & 2.68 & 1.32 \\
\hline $\begin{array}{l}\text { Inestabilidad } \\
\text { emocional }\end{array}$ & 2.02 & 1.51 & 1.32 & 1.31 & -0.70 & 1.75 & 1.74 & 1.25 & 1.74 & 1.22 & 0.00 & 1.64 & 0.67 & $2.96+$ & 2.46 \\
\hline $\begin{array}{l}\text { Elección compa- } \\
\text { ñero de juego }\end{array}$ & 4.85 & 2.29 & 8.53 & 3.20 & 3.68 & 3.57 & 6.37 & 3.10 & 6.63 & 2.43 & 0.26 & 2.69 & $6.15^{*}$ & $19.09^{* * * *}$ & $11.90^{* * *}$ \\
\hline $\begin{array}{l}\text { Rechazo compa- } \\
\text { ñero de juego }\end{array}$ & 2.66 & 2.40 & 2.13 & 2.32 & -0.53 & 2.60 & 2.67 & 1.71 & 2.70 & 3.13 & 0.04 & 2.63 & 0.00 & 0.83 & 1.00 \\
\hline $\begin{array}{l}\text { Elección compa- } \\
\text { ñero prosocial }\end{array}$ & 3.91 & 2.45 & 6.87 & 2.82 & 2.96 & 3.03 & 3.89 & 2.15 & 5.44 & 2.84 & 1.56 & 1.94 & 0.00 & $4.77^{*}$ & $5.60^{*}$ \\
\hline $\begin{array}{l}\text { Rechazo compa- } \\
\text { ñero prosocial }\end{array}$ & 2.17 & 2.20 & 2.23 & 2.43 & 0.06 & 2.32 & 2.96 & 1.67 & 2.52 & 2.32 & -0.44 & 2.02 & 2.68 & 0.90 & 0.12 \\
\hline Autoconcepto & 21.79 & 5.46 & 21.21 & 5.04 & -0.58 & 5.28 & 23.59 & 3.81 & 20.26 & 4.39 & -3.33 & 5.10 & 2.33 & $4.95^{*}$ & 2.62 \\
\hline $\begin{array}{l}\text { Personalidad } \\
\text { creadora } \\
\text { (profesores) }\end{array}$ & 34.06 & 10.70 & 43.58 & 12.52 & 9.53 & 11.80 & 36.70 & 12.75 & 40.56 & 12.46 & 3.85 & 8.53 & 0.96 & $4.91 *$ & $3.89+$ \\
\hline $\begin{array}{l}\text { Personalidad } \\
\text { creadora } \\
\text { (padres) }\end{array}$ & 38.13 & 8.01 & 40.51 & 9.96 & 2.38 & 8.70 & 40.26 & 8.22 & 40.44 & 11.20 & 0.19 & 9.92 & 1.23 & 1.03 & 0.52 \\
\hline
\end{tabular}


Los resultados obtenidos han puesto de relieve que este programa de educación para la convivencia ha estimulado significativamente cambios positivos en varios factores del desarrollo socio-emocional y creativo infantil (ver Tabla 2). En lo que se refiere a la socialización de los niños y niñas, los resultados del MANOVA pretest evidenciaron diferencias significativas entre experimentales y control, $F(1,78)=4.77$, $p<.001$, y también se encontraron diferencias en el MANCOVA pretest-postest, $F(1,78)=2.41, p<.05$. El análisis de cada variable de forma independiente (ANOVA) ratificó un incremento estadísticamente significativo de los experimentales frente a una disminución de los de control en jovialidad (experimentales $M=2.60$, control $M=-1.89), F(1,78)=6.73, p<.05$, sensibilidad social (experimentales $M=4.30$, control $M$ $=-2.41), F(1,78)=8.03, p<.01, \mathrm{y}$ respeto autocontrol (experimentales $M=5.21$, control $M=-1.85$ ), $F(1$, $78)=6.14, p<.05$. Por otro lado, los resultados del ANCOVA para aquellas variables en las que existían diferencias significativas entre ambas condiciones en la fase pretest, evidenciaron diferencias en ansiedadtimidez, en la que se observó una disminución en los experimentales $(M=-2.58)$ frente al incremento de los de control $(\mathrm{M}=3.63), F(1,78)=5.88, p<.05$.

En inestabilidad emocional, los resultados del ANOVA pretest no evidenciaron diferencias significativas entre ambas condiciones, $F(1,78)=0.67, p>$ .05. Sin embargo, el ANOVA pretest-postest muestra que los participantes experimentales disminuyeron en esta variable $(M=-0.70)$, en tanto que los de control se mantuvieron sin cambios $(M=0.00)$, si bien estas diferencias sólo fueron tendencialmente significativas, $F(1,78)=2.96, p<.10$.

En la elección/rechazo de niños y niñas como compañeros de juego y prosociales, el MANOVA pretest evidenció diferencias significativas entre experimentales y control, $F(1,78)=3.43, p<.05$, y también se encontraron diferencias en el MANCOVA pretestpostest, $F(1,78)=2.62, p<.05$. El análisis de cada variable de forma independiente (ANOVA) ratificó que los experimentales incrementaron significativamente la elección de compañeros prosociales $(M=2.96)$ frente a los de control $(M=1.56), F(1,78)=4.77, p<.05$. En el caso de la elección de compañero de juego, variable en la que existían diferencias significativas entre ambas condiciones en la fase pretest, el ANCOVA pretestpostest también evidenció un incremento significativo en los experimentales $(M=3.68)$ frente a los de control $(M=0.26), F(1,78)=11.90, p<.001$.
En el autoconcepto, el ANOVA pretest no evidenció diferencias significativas entre ambas condiciones, $F$ $(1,78)=2.33, p>.05$, en tanto que el ANOVA pretespostest mostró que las diferencias fueron estadísticamente significativas, con un descenso menor en los experimentales $(M=-0.58)$ frente a los de control $(M$ $=-3.33), F(1,78)=4.95, p<.05$.

Por último, en las conductas y rasgos de personalidad creadora, tomando conjuntamente la opinión de profesores y padres, los resultados del MANOVA pretest no evidenciaron diferencias significativas entre experimentales y control, $F(1,78)=0.79, p>.05$, en tanto que el MANOVA pretest-postest constató diferencias tendencialmente significativas entre ambas condiciones, $F(1,78)=2.56, p<.10$. Los ANOVAS de cada variable de forma independiente únicamente ratificaron diferencias estadísticamente significativas en el caso de la opinión de los profesores, $F(1,78)=4.91$, $p<.05$, con un aumento mayor en los experimentales $(M=9.53)$ que en los de control $(M=3.85)$.

\section{Desarrollo socio-emocional y creativo:} Diferencias en función del género

Con la finalidad de evaluar si existen diferencias significativas en las variables objeto de estudio en función del sexo se realizó un análisis de varianza con las puntuaciones pretest, cuyos resultados se presentan en la Tabla 3.

Los análisis de varianza (ver Tabla 3) evidenciaron puntuaciones significativamente superiores en las niñas en respeto-autocontrol (niños $M=31.39$, niñas $M=$ $40.29), F(1,78)=9.73, p<.01$, y adaptación social global (niños $M=31.08$, niñas $M=35.93), F(1,78)=$ $9.19, p<.01$. En lo referente a las conductas y rasgos de personalidad creadora, no se observaron diferencias de género en opinión de los padres, en tanto que, en opinión de los profesores, las niñas lograron puntuaciones significativamente superiores (niños $M=30.97$, niñas $M=38.55), F(1,78)=9.73, p<.01$.

Los niños puntuaciones bastante superiores en agresividad-terquedad (niños $M=9.00$, niñas $M=$ 5.05), $F(1,78)=4.67, p<.05$, apatía-retraimiento (niños $M=8.11$, niñas $M=4.38$ ), $F(1,78)=4.10$, $p<.05$, en rechazo de compañeros para jugar con ellos (niños $M=3.29$, niñas $M=2.10$ ), $F(1,78)=$ $6.35, p<.05$, y en rechazo a considerarlos prosociales (niños $M=3.63$, niñas $M=1.36), F(1,78)$ $=34.33, p<.001$. 
Tabla 3. Diferencias de género en variables socio-emocionales y de creatividad: medias, desviaciones típicas y resultados del ANOVA en la fase pretest.

\begin{tabular}{lcccccc}
\hline & \multicolumn{2}{c}{$\begin{array}{c}\text { Varón } \\
(n=38)\end{array}$} & \multicolumn{2}{c}{$\begin{array}{c}\text { Mujer } \\
(n=42)\end{array}$} & \multirow{2}{*}{$F(1,78)$} \\
\cline { 2 - 5 } Variables & $\mathrm{M}$ & $\mathrm{DT}$ & $\mathrm{M}$ & $\mathrm{DT}$ & \\
\hline Liderazgo & 21.63 & 11.87 & 26.50 & 11.25 & 3.54 \\
Jovialidad & 23.03 & 7.83 & 26.21 & 6.73 & 3.82 \\
Sensibilidad social & 20.50 & 10.25 & 24.60 & 8.28 & 3.89 \\
Respeto-autocontrol & 31.39 & 13.75 & 40.29 & 11.72 & $9.73^{* *}$ \\
Agresividad-terquedad & 9.00 & 9.12 & 5.05 & 7.20 & $4.67^{*}$ \\
Apatía-retraimiento & 8.11 & 9.30 & 4.38 & 7.07 & $4.10^{*}$ \\
Ansiedad-timidez & 8.84 & 5.62 & 8.40 & 4.84 & 0.14 \\
Adaptación social global & 31.08 & 8.58 & 35.93 & 5.53 & $9.19^{* *}$ \\
Inestabilidad emocional & 2.21 & 1.59 & 1.67 & 1.22 & 2.95 \\
Elección compañero de juego & 5.66 & 3.24 & 5.10 & 2.04 & 0.87 \\
Rechazo compañero de juego & 3.29 & 2.44 & 2.10 & 1.76 & $6.35^{*}$ \\
Elección compañero prosocial & 3.32 & 2.18 & 4.43 & 2.39 & 4.69 \\
Rechazo compañero prosocial & 3.63 & 2.14 & 1.36 & 1.24 & $34.33^{* * *}$ \\
Autoconcepto & 22.71 & 5.01 & 22.12 & 5.07 & 0.27 \\
Personalidad creadora (profesores) & 30.97 & 12.25 & 38.55 & 9.38 & $9.73^{* *}$ \\
Personalidad creadora (padres) & 38.34 & 6.98 & 39.31 & 9.04 & 0.28 \\
\hline
\end{tabular}

$* p<.05 * * p<.01 * * * p<.001$

\section{Influencia del género en los efectos del programa}

Con la finalidad de explorar si el programa tuvo un efecto diferencial con respecto al sexo, se llevaron a cabo análisis descriptivos (medias y desviaciones típicas) y de varianza (ANOVAs, ANCOVAs) en la fase pretest y en las diferencias pretest-postest en función del sexo, cuyos resultados se exponen en la Tabla 4.

Tomando en consideración las diferencias de género antes de la intervención, los ANCOVAs muestran únicamente que, tras la implementación del programa, las niñas eligieron significativamente más compañeros prosociales que los niños (niños $M=2.52$, niñas $M=$ $3.25), F(1,51)=7,13, p<.01$. Por tanto, cabe afirmar que el programa produjo pocos cambios diferenciales en función del género.

Percepción subjetiva del cambio en los sujetos experimentales: El cuestionario de evaluación del programa de educación para la convivencia

Con la finalidad de complementar los resultados de la evaluación experimental de la intervención, se exponen los resultados obtenidos al analizar los cuestionarios de evaluación del programa cumplimentados por los 53 participantes experimentales al finalizar dicha experiencia (ver Tabla 5).

En la Tabla 5 se puede observar la distribución de frecuencias y los porcentajes de respuesta del cambio percibido por los niños y niñas en diversos objetivos del programa de educación para la convivencia tras la finalización del mismo. Los participantes evalúan su nivel de cambio en relación a dichos objetivos, indicando si están "muy en desacuerdo", "en desacuerdo", "de acuerdo" o "muy de acuerdo" con las afirmaciones expresadas en las 17 afirmaciones presentadas. Como se puede observar, los 53 niños y niñas que participaron en el programa lo valoraron de forma muy positiva. Cabe resaltar que en todos los objetivos que contiene el cuestionario de evaluación indican que el nivel de cambio que han percibido en ellos mismos tras los tres años de implementación del programa es bastante grande.

En general, tal y como se muestra en la Tabla 5, más del $65 \%$ de los participantes se muestran de acuerdo o muy de acuerdo en lo referente a la percepción de 
Tabla 4. Efectos del programa de educación para la convivencia en variables socio-emocionales y de creatividad en función del género: medias, desviaciones típicas y resultados del ANOVA y ANCOVA para las diferencias pretest-postest.

\begin{tabular}{|c|c|c|c|c|c|c|c|c|c|c|c|c|c|c|c|}
\hline \multirow[b]{3}{*}{ Variables } & \multicolumn{6}{|c|}{$\begin{array}{c}\text { Varón } \\
(n=21)\end{array}$} & \multicolumn{6}{|c|}{$\begin{array}{c}\text { Mujer } \\
(n=32)\end{array}$} & \multicolumn{3}{|c|}{$\begin{array}{c}\text { Total } \\
(n=53)\end{array}$} \\
\hline & \multicolumn{2}{|c|}{$\begin{array}{l}\text { Evaluación } \\
\text { Pretest }\end{array}$} & \multicolumn{2}{|c|}{$\begin{array}{l}\text { Evaluación } \\
\text { Postest }\end{array}$} & \multicolumn{2}{|c|}{$\begin{array}{l}\text { Diferencia } \\
\text { Pre-Post }\end{array}$} & \multicolumn{2}{|c|}{$\begin{array}{c}\text { Evaluación } \\
\text { Pretest }\end{array}$} & \multicolumn{2}{|c|}{$\begin{array}{l}\text { Evaluación } \\
\text { Postest }\end{array}$} & \multicolumn{2}{|c|}{$\begin{array}{l}\text { Diferencia } \\
\text { Pre-Post }\end{array}$} & \multirow{2}{*}{$\begin{array}{c}\begin{array}{c}\text { Anova } \\
\text { Pre }\end{array} \\
F(1,51)\end{array}$} & \multirow{2}{*}{$\begin{array}{c}\begin{array}{c}\text { Anova } \\
\text { Pre-Post }\end{array} \\
F(1,51)\end{array}$} & \multirow{2}{*}{$\begin{array}{c}\begin{array}{c}\text { Ancova } \\
\text { Pre-Post }\end{array} \\
F(1,51)\end{array}$} \\
\hline & M & DT & M & DT & M & DT & M & DT & $M$ & $D T$ & $M$ & $D T$ & & & \\
\hline Liderazgo & 19.81 & 10.25 & 27.29 & 14.57 & 7.48 & 12.99 & 25.19 & 9.61 & 30.94 & 11.00 & 5.75 & 8.24 & 3.76 & 0.35 & 0.01 \\
\hline Jovialidad & 21.33 & 6.58 & 23.71 & 9.12 & 2.38 & 9.35 & 25.28 & 6.62 & 28.03 & 5.32 & 2.75 & 6.32 & $4.52 *$ & 0.29 & 1.99 \\
\hline Sensibilidad social & 17.52 & 7.35 & 23.52 & 9.59 & 6.00 & 10.56 & 23.63 & 6.04 & 26.81 & 6.16 & 3.19 & 7.53 & $10.86^{* *}$ & 1.28 & 0.45 \\
\hline Respeto-autocontrol & 26.05 & 11.61 & 34.24 & 11.62 & 8.19 & 10.24 & 39.41 & 11.78 & 42.66 & 8.19 & 3.25 & 12.02 & $16.47^{* * *}$ & 2.39 & 1.49 \\
\hline Agresividad-terquedad & 13.57 & 9.46 & 8.81 & 7.76 & -4.76 & 6.66 & 6.03 & 7.98 & 4.19 & 4.68 & -1.84 & 6.52 & $9.76^{* *}$ & 2.49 & 0.66 \\
\hline Apatía-retraimiento & 7.95 & 7.96 & 7.90 & 8.88 & -0.05 & 7.67 & 5.16 & 7.86 & 3.84 & 3.64 & -1.31 & 6.51 & 1.58 & 0.41 & 3.58 \\
\hline Ansiedad-timidez & 10.95 & 5.78 & 7.10 & 5.59 & -3.86 & 5.73 & 9.34 & 4.49 & 7.59 & 5.51 & -1.75 & 5.95 & 1.29 & 1.63 & 0.65 \\
\hline Adaptación social global & 29.00 & 7.62 & 32.43 & 8.79 & 3.43 & 6.47 & 35.44 & 5.38 & 37.78 & 4.03 & 2.34 & 6.14 & $1.98^{* * * *}$ & 0.37 & 1.43 \\
\hline Inestabilidad emocional & 2.38 & 1.74 & 1.90 & 1.54 & -0.48 & 1.77 & 1.78 & 1.31 & 0.94 & 0.98 & -0.84 & 1.74 & 2.03 & 0.55 & $6.23^{*}$ \\
\hline Elección compañero de juego & 4.67 & 2.57 & 7.71 & 3.08 & 3.05 & 3.51 & 4.97 & 2.13 & 9.06 & 3.21 & 4.09 & 3.61 & 0.21 & 1.08 & 2.07 \\
\hline Rechazo compañero de juego & 3.62 & 2.78 & 3.10 & 2.46 & -0.52 & 2.60 & 2.03 & 1.92 & 1.50 & 2.03 & -0.53 & 2.65 & $6.04^{*}$ & 0.00 & 3.09 \\
\hline Elección compañero prosocial & 2.86 & 2.19 & 5.38 & 2.80 & 2.52 & 2.69 & 4.59 & 2.40 & 7.84 & 2.41 & 3.25 & 3.25 & $7.05^{*}$ & 0.72 & $7.13^{* *}$ \\
\hline Rechazo compañero prosocial & 3.86 & 2.39 & 3.67 & 2.83 & -0.19 & 2.85 & 1.06 & 1.13 & 1.28 & 1.55 & 0.22 & 1.93 & $32.69^{* * *}$ & 0.38 & 3.42 \\
\hline Autoconcepto & 21.38 & 5.54 & 19.90 & 5.14 & -1.48 & 5.68 & 22.06 & 5.48 & 22.06 & 4.85 & 0.00 & 5.01 & 0.19 & 0.99 & 2.27 \\
\hline $\begin{array}{l}\text { Personalidad } \\
\text { creadora (profesores) }\end{array}$ & 29.38 & 12.13 & 39.76 & 13.05 & 10.38 & 10.80 & 37.13 & 8.52 & 46.09 & 11.69 & 8.97 & 12.55 & $7.46^{* *}$ & 0.17 & 0.41 \\
\hline $\begin{array}{l}\text { Personalidad } \\
\text { creadora (padres) }\end{array}$ & 37.52 & 7.45 & 40.14 & 9.76 & 2.62 & 8.99 & 38.53 & 8.45 & 40.75 & 10.23 & 2.22 & 8.65 & 0.19 & 0.02 & 0.00 \\
\hline
\end{tabular}

$* p<.05 * * p<.01 * * * p<.001$

cambios positivos relacionados con todos los objetivos del programa evaluados en el cuestionario. El objetivo sobre el que existe un mayor acuerdo en la percepción del cambio es el número 5, "Respeto más las opiniones de los/las demás" (el 90,6 \% de los niños y niñas están de acuerdo o muy de acuerdo con dicha afirmación), en tanto que menor acuerdo observado hace referencia al objetivo presentado en el ítem número 6 "Me atrevo a decir mis opiniones aunque no sean compartidas por los/las demás" (con todo, el 66 \% de los participantes están de acuerdo o muy de acuerdo con dicha afirmación).

\section{Discusión}

En lo referente a los efectos del programa en el desarrollo socio-emocional y creativo, los resultados sugieren que el programa de educación para la convivencia incrementó significativamente diversas conductas facilitadoras de la socialización (jovialidad, 
Tabla 5. Distribución de las frecuencias y porcentajes de respuesta del cambio percibido en objetivos de la intervención, valorados con el Cuestionario de Evaluación del programa de educación para la convivencia.

\begin{tabular}{|c|c|c|c|c|c|c|c|c|}
\hline & \multicolumn{2}{|c|}{$\begin{array}{c}\text { Muy en } \\
\text { desacuerdo }\end{array}$} & \multicolumn{2}{|c|}{$\begin{array}{c}\text { En } \\
\text { desacuerdo }\end{array}$} & \multicolumn{2}{|c|}{ De acuerdo } & \multicolumn{2}{|c|}{$\begin{array}{l}\text { Muy de } \\
\text { acuerdo }\end{array}$} \\
\hline & $\mathrm{n}$ & $\%$ & $\mathrm{n}$ & $\%$ & $\mathrm{n}$ & $\%$ & $\mathrm{n}$ & $\%$ \\
\hline 1. Participo más en actividades de grupo & 4 & $7.5 \%$ & 2 & $3.8 \%$ & 23 & $43.4 \%$ & 24 & $45.3 \%$ \\
\hline 2. Hablo más con mis compañeros/as de grupo & 4 & $7.5 \%$ & 2 & $3.8 \%$ & 17 & $32.1 \%$ & 30 & $56.6 \%$ \\
\hline 3. He hecho nuevos amigos/as dentro del grupo & 3 & $5.7 \%$ & 5 & $9.4 \%$ & 25 & $47.2 \%$ & 20 & $37.7 \%$ \\
\hline $\begin{array}{l}\text { 4. Me atrevo a decir mis opiniones aunque no sean } \\
\text { compartidas por los/las demás }\end{array}$ & 5 & $9.4 \%$ & 13 & $24.5 \%$ & 14 & $26.4 \%$ & 21 & $39.6 \%$ \\
\hline 5. Respeto más las opiniones de los/las demás & 3 & $5.7 \%$ & 2 & $3.8 \%$ & 30 & $56.6 \%$ & 18 & $34.0 \%$ \\
\hline 6. Estoy más atento a lo que necesitan mis compañeros/as & 5 & $9.4 \%$ & 3 & $5.7 \%$ & 24 & $45.3 \%$ & 21 & $39.6 \%$ \\
\hline 7. Comparto más mis cosas, mis juguetes... & 3 & $5.7 \%$ & 7 & $13.2 \%$ & 17 & $32.1 \%$ & 26 & $49.1 \%$ \\
\hline 8. Tengo más confianza en mis compañeros/as & 3 & $5.7 \%$ & 3 & $5.7 \%$ & 18 & $34.0 \%$ & 29 & $54.7 \%$ \\
\hline $\begin{array}{l}\text { 9. He aprendido a cooperar, a dar ayuda y a recibir ayuda } \\
\text { para realizar actividades de grupo }\end{array}$ & 4 & $7.5 \%$ & 4 & $7.5 \%$ & 24 & $45.3 \%$ & 21 & $39.6 \%$ \\
\hline 10. Expreso más lo que siento & 5 & $9.4 \%$ & 9 & $17.0 \%$ & 19 & $35.8 \%$ & 20 & $37.7 \%$ \\
\hline 11. Estoy más atento a los sentimientos de los/las demás & 2 & $3.8 \%$ & 5 & $9.4 \%$ & 17 & $32.1 \%$ & 29 & $54.7 \%$ \\
\hline 12. Me veo mejor a mí mismo/a, más bueno/a, valioso/a... & 5 & $9.4 \%$ & 4 & $7.5 \%$ & 25 & $47.2 \%$ & 19 & $35.8 \%$ \\
\hline $\begin{array}{l}\text { 13. Tengo mejor opinión de mis compañeros y compañeras, } \\
\text { creo que son valiosos/as, considerados/as, buenos/as... }\end{array}$ & 5 & $9.4 \%$ & 5 & $9.4 \%$ & 20 & $37.7 \%$ & 23 & $43.4 \%$ \\
\hline 14. Respeto más las reglas de los juegos & 6 & $11.3 \%$ & 0 & $0.0 \%$ & 22 & $41.5 \%$ & 25 & $47.2 \%$ \\
\hline $\begin{array}{l}\text { 15. Tengo ideas más originales y creativas cuando juego } \\
\text { con las palabras }\end{array}$ & 4 & $7.5 \%$ & 10 & $18.9 \%$ & 21 & $39.6 \%$ & 18 & $34.0 \%$ \\
\hline $\begin{array}{l}\text { 16. Tengo ideas más originales y creativas cuando dibujo, } \\
\text { pinto o hago trabajos manuales... }\end{array}$ & 4 & $7.5 \%$ & 6 & $11.3 \%$ & 14 & $26.4 \%$ & 29 & $54.7 \%$ \\
\hline $\begin{array}{l}\text { 17. Me han gustado mucho las actividades que hemos } \\
\text { realizado durante estos tres años, han sido muy divertidas }\end{array}$ & 2 & $3.8 \%$ & 5 & $9.4 \%$ & 23 & $43.4 \%$ & 23 & $43.4 \%$ \\
\hline
\end{tabular}

sensibilidad social, respeto-autocontrol), el número de compañeros de juego y de compañeros percibidos como prosociales, y las conductas-rasgos de personalidad creadora en opinión de los profesores. Además, se observa que el autoconcepto, pese a disminuir en ambas condiciones experimentales, lo hizo significativamente menos en el grupo experimental que en el de control. Por tanto, se ratifica la primera hipótesis prácticamente en su totalidad, ya que únicamente no se observaron diferencias significativas en lo relativo a liderazgo, adaptación social global y conductas y rasgos de personalidad creadora en opinión de los padres.

Por otro lado, los resultados evidencian que el programa disminuyó significativamente la ansiedadtimidez de los participantes $\mathrm{y}$, tendencialmente, su inestabilidad emocional, lo que nos lleva a ratificar parcialmente la segunda hipótesis, ya que no se observó una disminución en otras conductas perturbadoras de la socialización (agresividad-terquedad, apatíaretraimiento), en el rechazo de compañeros de juego, 
ni en el número de compañeros percibidos como no prosociales. Además, los resultados del cuestionario de evaluación del programa, administrado a los experimentales al finalizar la intervención, apuntan en la misma dirección que los resultados de la evaluación experimental, ya que muestran, desde la percepción subjetiva de los implicados, un cambio positivo en diversos factores conductuales y cognitivos de la personalidad infantil.

Los resultados de en este estudio confirman los obtenidos en otros estudios que también han constatado los efectos positivos que tienen los programas de intervención psicoeducativa basados en actividades cooperativas y creativas en el desarrollo socio-emocional y creativo infantil (Garaigordobil 2003ab, 2004ab, 2005ab; Garaigordobil \& Fagoaga, 2006; Garaigordobil \& Pérez, 2004). Los resultados han evidenciado un aumento de las conductas sociales positivas y disminución de las negativas, permitiendo sugerir que este tipo de experiencias pueden ser eficaces para prevenir la violencia, tal y como se ha constatado en otros estudios (Chandy, 2007; Dawn \& Shaughnessy, 2005; Dole, 2006; Farrell, Meyer \& White 2001; Freiden, 2006; Roberts, White \& Yeomans, 2004; Segawa, Ngwe, Li \& Flay, 2005; Sege, Licenziato \& Webb, 2005; Simón et al., 2008; Slone \& Shoshani, 2008).

En lo que respecta a las diferencias de género en el desarrollo socio-emocional y creativo, subrayar que antes de la implementación del programa se observaron diferencias de género significativas en diversas variables. Los análisis de varianza evidenciaron puntuaciones significativamente superiores en las niñas en respeto-autocontrol y adaptación social global, lo que permite ratificar parcialmente la tercera hipótesis. Los niños obtuvieron puntuaciones significativamente superiores en agresividad-terquedad, apatía-retraimiento, y en el rechazo de compañeros para jugar con ellos o considerarlos prosociales. Por consiguiente, se ratifica la cuarta hipótesis prácticamente en su totalidad. No se observaron diferencias de género en autoconcepto, lo que confirma la quinta hipótesis. Por último, en lo que a diferencias de género se refiere, no se observaron diferencias en lo referente a las conductas y rasgos de personalidad creadora en opinión de los padres, en tanto que, en opinión de los profesores, las niñas alcanzarón puntuaciones significativamente superiores. Por consiguiente, la sexta hipótesis de este estudio se ratifica tan solo parcialmente.

Respecto a la influencia del género en los efectos del programa, los resultados confirman que el programa de educación para la convivencia produjo pocos cambios diferenciales en función del género. Únicamente se observa que, tras la implementación del programa, las niñas eligieron significativamente más compañeros prosociales que los niños. Consiguientemente, la séptima hipótesis, que planteaba que el género no afectaría a los efectos del programa de educación para la convivencia, se ratifica prácticamente en su totalidad. Los resultados apuntan en la misma dirección que otros estudios que apenas evidencian efectos diferenciales en función del género en este tipo de intervenciones (Garaigordobil \& Fagoaga, 2006; Garaigordobil \& Pérez, 2004).

Los datos en su conjunto ratifican el efecto positivo de las intervenciones basadas en las actividades cooperativas y creativas, y validan un programa de intervención psicopedagógica para Educación Primaria de tres años de duración que, mediante el empleo de diversas técnicas, materiales y soportes comunicativos, estimula el desarrollo socio-emocional y creativo de niños y niñas en el marco de la educación en la convivencia, el respeto de los derechos humanos y la cultura de paz. Por otra parte, este trabajo enfatiza la existencia de diferencias de género, y evidencia mayores niveles de adaptación socio-emocional en las niñas, en tanto que los niños muestran un mayor número de factores negativos o perturbadores de la misma.

La explicación a estas diferencias de género no está del todo clara, si bien pudieran atribuirse a las diferencias existentes en la educación y patrones de socialización de niños y niñas. Se podría asumir que en general las niñas, al menos en los países occidentales, tienden a ser socializadas de forma tal que se favorecen en mayor medida que en los niños las relaciones interpersonales positivas. La aceptación de la violencia en la sociedad se relaciona con valores culturales que consideran la violencia como una forma natural de solucionar los conflictos, particularmente entre los varones. Se asocia la resolución de conflictos mediante el lenguaje, el diálogo y respuestas empáticas con las mujeres, en tanto que en el caso de los varones se tolera en mayor medida las respuestas agresivas físicas y verbales frente a los modelos de autoridad y poder. Los resultados de este estudio nos llevan a sugerir la necesidad de reflexionar y modificar el tipo de educación y patrones de socialización que se promueven en el caso de los niños, puesto que conllevan mayores dificultades para su adaptación socio-emocional. Asimismo, estos resultados sugieren la necesidad de analizar críticamente los modelos de conducta asociados al género que se ofrecen a los niños y niñas desde los medios de comunicación (TV, Internet, revistas, etc.). 
El estudio deja abiertas futuras líneas de investigación, tales como: (1) la exploración de los efectos del programa en el desarrollo cognitivo, o en otras variables del desarrollo socio, emocional y creativo; (2) la evaluación del efecto del programa en interacción con las características del adulto que dirige la intervención; y (3) la utilización de otras estrategias de evaluación, como por ejemplo, técnicas observacionales para medir las variables dependientes.

\section{Referencias}

Chandy, S. R. (2007). Best practices and positive youth development program evaluation of a parenting-based youth violence prevention program. Dissertation Abstracts International: Section B: The Sciences and Engineering, 67(7-B), 4098.

Dawn, J., \& Shaughnessy, J. (2005). Promoting non-violence in schools: the role of cultural, organisational and managerial factors. Educational and Child Psychology, 22, 58-66.

Dole, K. (2006). The effect of the Balance Program on aggression in the classroom. Dissertation Abstracts International: Section B: The Sciences and Engineering, 67(2-B), 1180.

Farrell, A. D., Meyer, A. L., \& White, K. S. (2001). Evaluation of Responding in Peaceful and Positive Ways (RIPP): a schoolbased prevention program for reducing violence among urban adolescents. Journal of Clinical Child Psychology, 30, 451-463.

Freiden, J. (2006). Game: a clinical intervention to reduce adolescent violence in schools. Dissertation Abstracts International Section A: Humanities and Social Sciences, 66(12-A), 4308.

Garaigordobil, M. (2003a). Intervención psicológica para desarrollar la personalidad infantil. Juego, conducta prosocial y creatividad. Madrid: Pirámide (volumen 1).

Garaigordobil, M. (2003b). Programa Juego 8-10 años. Juegos cooperativos y creativos para grupos de niños de 8 a 10 años. Madrid: Pirámide (volumen 3).

Garaigordobil, M. (2004a). Programa Juego 10-12 años. Juegos cooperativos y creativos para grupos de niños de 10 a 12 años. Madrid: Pirámide (volumen 4).

Garaigordobil, M. (2004b). Intervención psicológica en la conducta agresiva y antisocial con niños. Psicothema, 16(3), 429-435.

Garaigordobil, M. (2005a). Programa Juego 6-8 años. Juegos cooperativos y creativos para grupos de niños de 6 a 8 años. Madrid: Pirámide (volumen 2).
Garaigordobil, M. (2005b). Prosocial and creative play: Effects of a program on the verbal and nonverbal intelligence of children aged 10-11 years. International Journal of Psychology, 40(3), 176-188.

Garaigordobil, M., \& Fagoaga, J. M. (2006). Juego cooperativo para prevenir la violencia en los centros educativos: Evaluación de programas de intervención para educación infantil, primaria y secundaria. Madrid: Ministerio de Educación y Ciencia. Dirección general de Educación, Formación Profesional e Innovación Educativa. Centro de Investigación y Documentación Educativa.

Garaigordobil, M., \& Pérez, J. I. (2004). Evaluación de un programa de juego creativo: Implicaciones en la estabilidad emocional, las estrategias de interacción social y el autoconcepto de niños de 10 a 11 años. Educación y Ciencia, 8(15), 65-84.

Garaigordobil, M., \& Pérez, J. I. (2005). Escala de Personalidad Creadora: Estudio psicométrico exploratorio. Estudios de Psicología, 26(3), 345-364.

Koppitz, E. (1976). El dibujo de la figura humana en los niños. Buenos Aires: Guadalupe (trabajo original publicado en 1968).

Moreno, J. L. (1972). Fundamentos de la sociometría. Buenos Aires: Paidós (trabajo original publicado en 1934).

Roberts, L., White, G., \& Yeomans, P. (2004). Theory development and evaluation of project WIN: a violence reduction program for early adolescents. The Journal of Early Adolescence, 24, 460-483.

Segawa, E., Ngwe, J. E., Li, Y., \& Flay, B. R. (2005). Evaluation of the effects of the Aban Aya Youth Project in reducing violence among African American adolescent males using. Evaluation Review, 29, 128-148.

Sege, R. D., Licenziato, V. G., \& Webb, S. (2005). Bringing violence prevention into the clinic: the Massachusetts medical society violence prevention project. American Journal of Preventive Medicine, 29, 230-232.

Silva, F., \& Martorell, M.C. (1983). Batería de socialización BAS 1 y 2. Madrid: TEA.

Simon, T. R., Ikeda, R. M., Smith, E. P., Reese, L. E., Rabiner, D. L., Miller-Johnson, S., Winn, D. M., Dodge, K. A., Asher, S. R., Home, A. M., Orpinas, P., Martin, R., Quinn, W. H., Tolan, P. H., Gorman-Smith, D., Henry, D. B., Gay, F. N., Schoeny, M., Farrell, A. D., Meyer, A. L., Sullivan, T. N., \& Allison, K. W. (2008). The Multisite Violence Prevention Project: impact of a universal school-based violence prevention program on social-cognitive outcomes. Prevention Science, 9, 231-244.

Slone, M., \& Shoshani, A. (2008). Efficacy of a school-based primary prevention program for coping with exposure to political violence. International Journal of Behavioral Development, 32, 348-358.

UNICEF (2006). Convención de los derechos del niño. Madrid: UNICEF Comité Español (trabajo original publicado en 1989). 